\title{
Predictors of Early and Late Escalation of Smokeless Tobacco Use and Cigarette Smoking Among Swedish Adolescents
}

\author{
K. Ingvar Rosendahl ${ }^{*}, 1,2$ and M. Rosaria Galanti ${ }^{1}$ \\ ${ }^{I}$ Division of Public Health Epidemiology, Department of Public Health Sciences, Karolinska Institutet, Stockholm, \\ Sweden \\ ${ }^{2}$ Centre for Psychiatry Research, Department of Clinical Neuroscience, Karolinska Institutet, Stockholm, Sweden
}

\begin{abstract}
Knowledge concerning developmental trajectories of tobacco use has until recently been limited to smoking. We utilized information on 960 adolescents who participated in the BROMS cohort study, with follow-up between the ages of 11 and 18 years. Factors related to personal monetary allowance, psychosocial factors and alcohol drinking were used to predict individual probability of progression of snus (the Swedish smokeless tobacco) and of cigarettes. Predictors of snus use and smoking showed more similarities than differences. Friends' and parents' tobacco use did only discriminate between early and late progression in cigarette smoking $(\mathrm{OR}=3.2, \mathrm{CI}=1.2-8.7$ for friends' tobacco use and $\mathrm{OR}=1.5, \mathrm{CI}=1.0-2.1$ for parents' tobacco use). Weekly alcohol drinking was the strongest predictor of early progression to use tobacco $(\mathrm{OR}=2.6, \mathrm{CI}=1.1-6.2$ for snus use and $\mathrm{OR}=6.3, \mathrm{CI}=2.4-16.8$ for cigarette smoking). This knowledge can be used as a base for dedicated prevention strategies targeting different groups of young users.
\end{abstract}

Keywords: Smokeless tobacco, cigarette smoking, tobacco use, trajectories, adolescents, longitudinal.

\section{INTRODUCTION}

The study of behavioral changes over the span of the life course is an appropriate strategy to identify groups or time periods of particular susceptibility to transitioning to use drugs. Concerning adolescent smoking, results from longitudinal studies are not easily comparable. However, two general patterns are discernible: stability and escalations of different intensities [1,2]. Studies of smoking behavior have also addressed risk factors for belonging to different developmental trajectories. Among others, gender [2], poor academic performance, behavior problems, anxiety [2, 4-6]; family and peer factors [2-6]; intentions and beliefs $[3,6]$ have been considered. Also, smoking seems to coincide with the use of other substances [4-6]. As a whole, these findings confirm analytical studies of risk factors for smoking among adolescents. In contrast to cigarette smoking, long-term patterns of adolescent smokeless tobacco use have not been investigated until recently, in the frame of a longitudinal study of Swedish adolescents [7]. Using information from the same cohort study, we conducted the present analysis in order to compare escalating trajectories of smokeless tobacco use and of cigarette smoking in adolescence according to predictors in the following domains: personal monetary allowance, that was compared to youth smoking in etiological studies [8], psychosocial factors and alcohol drinking which is the most frequent substance of abuse among Swedish adolescents.

*Address correspondence to this author at the STAD Centre for Psychiatry Research, Stockholm County Council/Karolinska Institutet, P.O. Box 6031, SE-102 31 Stockholm, Sweden; Tel: +46 8123455 14; Fax: +46 8123455 09; E-mail: ingvar.rosendahl@ki.se

\section{MATERIALS AND METHODOLOGY}

\section{Subjects}

The BROMS (Swedish acronym for Children's Smoking and Environment in the Stockholm County) cohort encompasses 3020 children which were recruited in 1997, at the time when they attended $5^{\text {th }}$ grade ( 11 years of age). The details of the recruitment of the cohort have been described previously $[9,10]$. Members in this cohort were followed up to the age of 18. During the study time, 960 of these adolescents, who reported never to have used tobacco at baseline, escalated tobacco use within the trial during an early or late stage [7], and constitute the study sample in this analysis.

\section{Information on Tobacco Use}

After the baseline survey conducted at the age 11, six follow-up surveys were conducted during the study period (at ages 12-15, 17 and 18 years). Information was collected by means of a self-administered questionnaire, with separate but very similar questions on past and current use of snus (the Swedish variety of smokeless tobacco) and cigarettes. An index of yearly consumption of snus portions and/or cigarettes was estimated based on these reports. This index was then used to estimate the number and shapes of developmental trajectories of use by using a semi-parametric group-based modeling (TRAJ) [7]. For both types of tobacco two trajectories indicating progression of tobacco use were identified in a previous study [7], peaking at the age of 15 years (early escalation) and at the age of 17 years (late escalation), respectively. Escalation of tobacco use was defined as any sustained increase in consumption at followup compared to baseline. Early and late escalation was judged on a relative time scale. 


\section{Information on Predictors of Tobacco Use}

At each survey information on several putative risk factors for tobacco use was collected from the adolescents or their parents. For the purpose of this study we focused on the following predictors: a) personal monetary allowance availability of pocket-money (weekly/monthly from parents in compensation for home service; weekly/monthly from the parents free of obligations; from parents whenever needed; through paid work outside home; the entire monthly government child subsidy given to the family; no personal income) was dichotomized into "availability as compensation for service within the household" $v s$ "free of obligations" (26 students had no pocket-money at all and were treated as missing information on this variable); amount of pocket-money converted into United States Dollars (USD) available per week $(<3 ; 3-8 ; 8-15 ; 15$ $31 ;>31)$ and dichotomized at $\$ 8$ or more $v s$ less than $\$ 8$ per week. b) psychosocial factors and psychosocial influences whether the adolescent would rather be someone else than self (yes/don't know vs no); friends' and parents' tobacco use which was dichotomized as "at least one" using any tobacco vs "none"; occurrence of any of the following stressful life-events during the previous year: moved to a new address; moved to a new school; divorce/separation in the family; death of a relative; parents' unemployment. A cumulative count variable ( 0 to 5 ) for these events was then dichotomized as "more than one event" vs "one or none". c) alcohol drinking - categorized as weekly vs less than weekly drinking of any alcoholic beverage at the age of 13 years (including beer $2.5-3.5 \%$ alcohol by volume; beer $>3.5 \%$ alcohol by volume; wine; liquor). In order to rule out potential confounding factors due to social circumstances we also included the following socio-demographic information in the analysis: student's country of origin (Sweden or outside Sweden); parents' country of origin (Sweden vs outside Sweden or unknown); parents living together (yes/no); parents' education (number of completed years of education). A dichotomous variable indicating parents' educational level corresponding to college level vs less than college level was created for the purpose of the analysis primarily using primarily mother's education, secondly father's education, and thirdly the educational level required for the employment in the current job position ( 2 percent of the subjects). Information on parents' profession, education, and history of tobacco use was collected at baseline through a parents' survey.

\section{Data Analysis and Statistical Methods}

The outcome variable in the present analysis was the probability of belonging to the most advanced trajectory group of snus use or cigarettes [7]. Binary logistic regression models were used to derive the odds ratios (OR) and corresponding $95 \%$ confidence intervals (CI) of early escalation vs late escalation of snus use, and of cigarette smoking. Socio-demographic factors were used in the analysis to adjust for potential confounders and were associated differently to snus use and cigarette smoking in the univariate analyses. Therefore, factors included for adjustment in multivariate models presented in Table $\mathbf{2}$ may differ. All multivariate analyses were performed for the whole sample and separated by gender.

\section{RESULTS}

Table 1 presents the prevalence (\%) of predictors measured in the $5^{\text {th }}$ grade (socio-demographic, personal monetary allowance, and psychosocial predictors) or in the

Table 1. Prevalence (\%) of Predictors in Escalation Trajectories of Tobacco Use

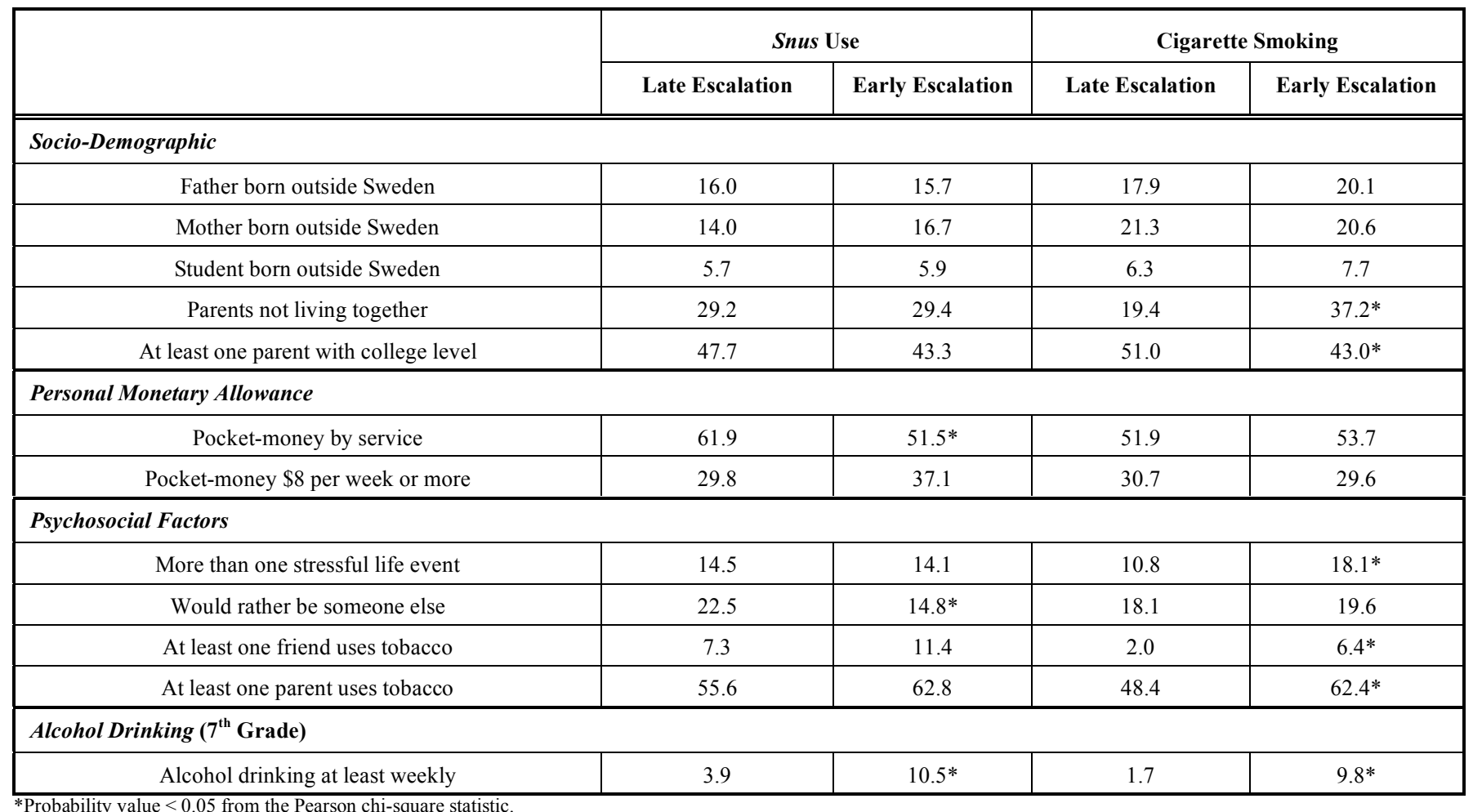


$7^{\text {th }}$ grade (alcohol drinking) in subgroups defined by developmental trajectories of snus use and smoking.

In a bivariate analysis (Table 1) socio-demographic factors did not differ statistically between the trajectories of snus use, while parents not living together as well as parents not having a college level education were overrepresented in the early escalation group of cigarette smoking compared to the late escalation group. High availability of pocket-money did not differ between the trajectories for either snus use or cigarette smoking, even though absence of a compensation system were overrepresented in the early escalating consumption trajectory of snus use. Most psychosocial predictors were unequally distributed between the early and late escalating consumption trajectories of cigarette use, with a higher prevalence in the early escalating trajectory. In contrast to cigarette smoking, "would rather be someone else" as a measure of low self-esteem was the only psychosocial factor unequally distributed between the early and late escalation trajectory of snus use. Moreover, it was also unequally distributed in the opposite direction compared to the psychosocial factors found significant for smoking trajectories, with a lower prevalence among the early escalation trajectory of snus. The only factor discriminating between the early and late escalation group for both tobacco products was own alcohol drinking. Table $\mathbf{2}$ displays OR for snus use (left side) and for cigarette smoking (right side), comparing the probability of being in the early escalation group vs late escalation group.

Personal monetary allowance, multiple stressful life events and low self-esteem were no longer statistically significant after adjustment for potential confounders. For cigarette smoking, tobacco use among at least one friend or at least one parent remained significantly associated to early escalation, although friends' tobacco use was only significant among boys and parents' tobacco use only among girls. The only predictor that remained significantly associated to early escalation for both tobacco products was weekly alcohol drinking at the age of 13 years. However, the association with alcohol drinking was weaker for snus use than for cigarette smoking, and only statistically significant among boys. The analysis separated by gender revealed that alcohol drinking was a stronger predictor of early escalation among boys than it was among girls.

\section{DISCUSSION}

A pioneer study of snus use compared to cigarette smoking, using data from this cohort indicated that the developmental trajectories of these behaviors have a similar shape and share most known risk factors for tobacco use among youths [7]. This is consistent with studies documenting similarities between trajectories of substance use among adolescents $[11,12]$. The purpose of the present study was to advance this knowledge by investigating predictors of early escalation of tobacco, a behavior that is of utmost concern for prevention. In fact, universal prevention may be more successful in delaying progression rather than in avoiding any initiation of use [1]. We hypothesized that, apart from social influences [5], other risk factors triggering escalation could be traced in the following domains: availability of money, psychosocial factors and other substance use, particularly alcohol drinking. Specifically, we were interested in understanding whether indicators in these domains equally predict early escalation of snus use and of cigarette smoking.

\section{Personal Monetary Allowance}

Several studies have provided evidence that increasing cigarette prices reduces smoking among adolescents and young adults, who are particularly price-sensitive [13]. Some evidence also suggests that boys may be more sensitive to price increases than girls [8]. We hypothesized that availability of money to adolescents could be implicated not only in smoking initiation but also in the progression of both smokeless tobacco use and cigarette smoking. Further, we hypothesized that availability of money would be a weaker predictor of escalation of snus use than of cigarette smoking since the current Swedish retail price of snus is much lower than that of cigarettes. Our data did not support these hypotheses. In contrast to Scragg R, et al. [14] who found a positive relation between amount of pocket-money and cigarette smoking in adolescents, and to Mohan S et al. [15] who found the same relationship with all tobacco use, we did not find any statistically significant relation between availability of money and escalation of tobacco use. If anything, factors pertaining to personal monetary allowance were associated with escalation of snus use rather than cigarette smoking, at least among boys. In fact, availability of pocket-money exceeding the Swedish standard for an 11year old in 1998 was linked to early escalation with snus, while effort needed to gain money (e.g. through service at home) was linked to a decreased probability of escalating snus use. There may be several explanations for this counterintuitive pattern of association. First, money availability in the pre-teens may predict early experimental use but not progression. In order to fully understand the role that personal monetary allowance plays in the progression of tobacco use, it would have been necessary to track availability of money during the whole course of adolescence. Second, a law prohibiting sales of tobacco to minors was issued in 1997, the year the cohort was accrued. This prohibition may have played down the role of money availability as a risk factor for adolescents purchasing tobacco. However, it is known that the retailers' compliance with the law is not satisfactory, since $60 \%$ of 16 -year old smokers in Sweden reported buying their own cigarettes even after the law was enforced [16]. Besides, compliance with the law could only prevent the direct trade between underage and tobacco retailers, not the person- to person trade. A third plausible explanation is the residual confounding factor of socio-economic status of the family, of which pocket money may be an indicator.

\section{Psychosocial Factors}

Psychosocial factors may be relevant in identifying subgroups of high-risk adolescents [17], since early escalation of tobacco use most likely reflects individual vulnerability to substance use. Friends' and parents' smoking were investigated as correlates of adolescents' smoking trajectories in two previous studies [3, 4]. In our study, exposure to social influences of friends' and parents' tobacco use did differentiate between early and late escalation of smoking as seen in the studies by Chassin et al. and Orlando et al. However, friends' tobacco use only influenced boys 
Table 2. OR and $95 \%$ CI of Belonging to the Early Escalating Group Compared to the Late Escalating Group

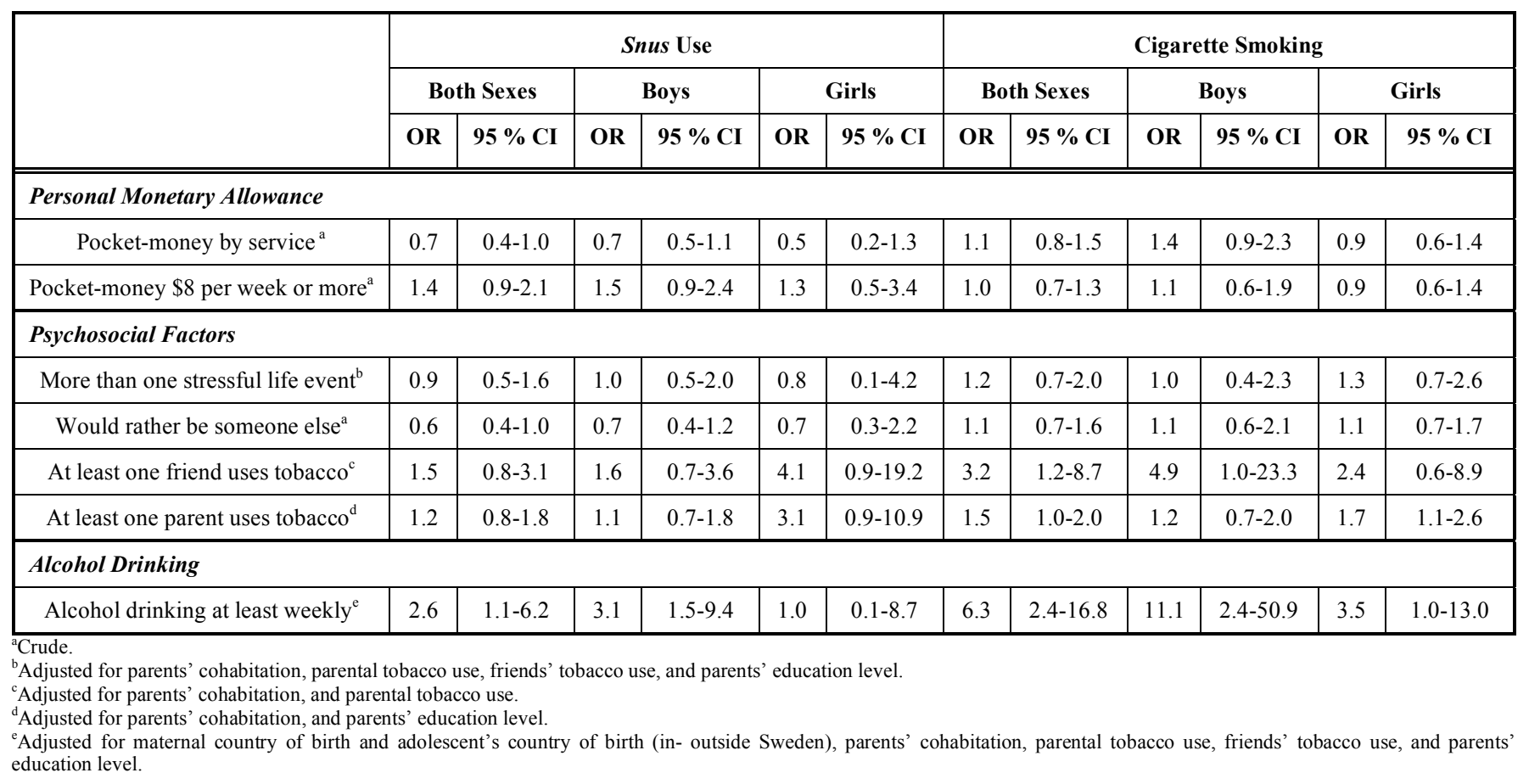

and parents' tobacco use only influenced girls. There are two main differences between the studies by Chassin et al. and Orlando et al. and this study. First, we included both current smokeless tobacco use and smoking among parents and friends, while previous studies only measured smoking. Second, parental smoking was assessed through adolescents' reports, while in our study tobacco use was reported from the parents themselves. Neither friends' nor parents' tobacco use differed between the trajectories of snus use. Both selfesteem and psychological well-being have previously been associated with initiation of both cigarettes and snus $[9,18$, 19]. In our study, neither stressful life events nor low selfesteem (expressed as the wish to be someone else rather than self) discriminated between early and late progression of tobacco use.

\section{Alcohol Drinking}

Alcohol drinking was addressed in this study in order to represent general vulnerability to substance use. In line with the study by Orlando et al. [12] we found that alcohol use was indeed a strong predictor of progression of tobacco use in this sample. Drinking alcohol at least weekly at the age of 13 was associated with an increased probability to belong to the group with early escalation of snus use among boys as well as of cigarette consumption in both sexes. Thus, the data supported our a-priori hypothesis that adolescents showing the most explosive development of tobacco use are more prone to be multi-drug users, and this may reflect underlying genetic and/or environmental aspects [1]. While the pattern alcohol-smoking escalation was common among both genders, some differences emerged in the strength of this association, where this was more pronounced among boys than among girls. This finding is in line with patterns of alcohol consumption in the general adolescent population of Sweden, with higher prevalence and earlier onset among males [16].

\section{Strengths and Weaknesses}

Strengths of the study include the large sample size, the longitudinal design and high retention rate. An important limitation in this study was that most predictors were assessed at different points in time during the course of adolescence, without subsequent re-assessment. It is likely that the impact of some explanatory factors may vary over time, i.e. according to age or stage of tobacco use.

\section{CONCLUSION}

Alcohol drinking was a strong predictor of progression of tobacco use in both genders. Social influences of tobacco use by friends and parents do seem to be of importance, although with a gender difference, in anticipating escalation of cigarette smoking while it did not seem to be of importance for snus use. Our findings add to previous observations that adolescents exhibiting a rapid escalation of drug use are a vulnerable group that should be targeted by dedicated prevention programs which address all substance use.

\section{ACKNOWLEDGEMENTS}

We wish to thank the BROMS study team for devoted retaining of the study cohort and Sara Sanchez for language review. The BROMS Cohort Study was funded by The Stockholm Centre for Public Health - Tobacco Prevention, Stockholm County Council, and by The Swedish Research Council, grant no. 345-2002-35.

\section{REFERENCES}

[1] Colder CR, Mehta P, Balanda K, et al. Identifying trajectories of adolescent smoking: an application of latent growth mixture modeling. Health Psychol 2001; 20(2): 127-35.

[2] Karp I, O'Loughlin J, Paradis G, Hanley J, Difranza J. Smoking trajectories of adolescent novice smokers in a longitudinal study of tobacco use. Ann Epidemiol 2005; 15(6): 445-52.

[3] Chassin L, Presson CC, Pitts SC, Sherman SJ. The natural history of cigarette smoking from adolescence to adulthood in a 
Midwestern community sample: multiple trajectories and their psychosocial correlates. Health Psychol 2000; 19(3): 223-31.

[4] Orlando M, Tucker JS, Ellickson PL, Klein DJ. Developmental trajectories of cigarette smoking and their correlates from early adolescence to young adulthood. J Consult Clin Psychol 2004; 72(3): 400-10.

[5] Audrain-McGovern J, Rodriguez D, Tercyak KP, Cuevas J, Rodgers K, Patterson F. Identifying and characterizing adolescent smoking trajectories. Cancer Epidemiol Biomarkers Prev 2004; 13(12): 2023-34.

[6] Stanton WR, Flay BR, Colder CR, Mehta P. Identifying and predicting adolescent smokers' developmental trajectories. Nicotine Tob Res 2004; 6(5): 843-52.

[7] Rosendahl KI, Galanti M. Rosaria, Gilljam H. Trajectories of smokeless tobacco use and of cigarette smoking in a cohort of Swedish adolescents: differences and implications. Nicotine Tob Res 2008; 10(6): 1021-7.

[8] Thomas S, Fayter D, Misso K, Ogilvie D, Petticrew M, Sowden A, et al. Population tobacco control interventions and their effects on social inequalities in smoking: systematic review. Tob Control 2008; 17(4): 230-7.

[9] Galanti MR, Rosendahl I, Post A, Gilljam H. Early gender differences in adolescent tobacco use--the experience of a Swedish cohort. Scand J Public Health 2001; 29(4): 314-7.

[10] Post A, Galanti MR, Gilliam H. School and family participation in a longitudinal study of tobacco use: some methodological notes. Eur J Public Health 2003; 13(1): 75-6.
[11] Duncan SC, Duncan TE, Biglan A, Ary D. Contributions of the social context to the development of adolescent substance use: a multivariate latent growth modeling approach. Drug Alcohol Depend 1998; 50(1): 57-71.

[12] Orlando M, Tucker JS, Ellickson PL, Klein DJ. Concurrent use of alcohol and cigarettes from adolescence to young adulthood: an examination of developmental trajectories and outcomes. Subst Use Misuse 2005; 40(8): 1051-69.

[13] Forster JL, Widome R, Bernat DH. Policy interventions and surveillance as strategies to prevent tobacco use in adolescents and young adults. Am J Prevent Med 2007; 33(6 Suppl): S335-9.

[14] Scragg R, Laugesen M, Robinson E. Cigarette smoking, pocket money and socioeconomic status: results from a national survey of 4th form students in 2000. N Z Med J 2002; 115(1158): U108.

[15] Mohan S, Sankara SP, Thankappan KR. Access to pocket money and low educational performance predict tobacco use among adolescent boys in Kerala, India. Prev Med 2005; 41(2): 685-92.

[16] Hvitfeldt T, Rask L. Skolevlevers drogvanor 2005. Rapport $\mathrm{nr} 90$ 2005 Available from: http: //www.can.se [cited: May 20, 2008].

[17] Galanti MR, Rosendahl I, Wickholm S. The development of tobacco use in adolescence among "snus starters" and "cigarette starters": an analysis of the Swedish "BROMS" cohort. Nicotine Tob Res 2008; 10(2): 315-23.

[18] Conrad KM, Flay BR, Hill D. Why children start smoking cigarettes: predictors of onset. Br J Addict 1992; 87(12): 1711-24.

[19] Wills TA, Shiffman S. Coping and substance use: a conceptual framework. New York: Academic Press 1985.

(C) Rosendahl and Galanti; Licensee Bentham Open.

This is an open access article licensed under the terms of the Creative Commons Attribution Non-Commercial License (http://creativecommons.org/licenses/by$\mathrm{nc} / 3.0 /$ ) which permits unrestricted, non-commercial use, distribution and reproduction in any medium, provided the work is properly cited. 